\title{
Multiplicity difference correlators under first-order QGP phase transition
}

\author{
C.B. Yang ${ }^{1,2}$ and X. Cai ${ }^{1}$ \\ 1 Institute of Particle Physics, Hua-Zhong Normal University, Wuhan 430079, P.R. China \\ 2 Theory Division, RMKI, KFKI, Budapest 114., Pf. 49, H-1525 Hungary
}

(August 10, 2018)

\begin{abstract}
The multiplicity difference correlators between two well-separated bins in high-energy heavy-ion collisions are studied as a means to detect evidence of a first-order quark-hadron phase transition. Analytical expressions for the scaled factorial moments of multiplicity difference distribution are obtained for small bin size $\delta$ with mean multiplicity in the bin $\bar{s} \leq 1.0$ within Ginzburg-Landau description. The scaling behaviors between the moments are still valid, though they behave completely different from the so-called intermittency patterns. A universal exponent $\gamma=1.4066$ is given to describe the dynamical fluctuations in the phase transition in small $\delta$ limit.
\end{abstract}

The theoretical study of fluctuations in quark-hadron phase transition has been suggested for several years ${ }^{[1]}$. The first motivation came from the quantitatively different results in Monte Carlo simulations ${ }^{[2]}$ on intermittency for $p p$ collisions without phase transition from those theoretical predictions with the onset of phase transition ${ }^{[1]}$. Since then, multiplicity fluctuations are studied with phase transitions of second-order ${ }^{[3]}$ and first-order ${ }^{[4-6]}$ within GinzburgLandau model, and it is regarded as a possible means to reveal some features for the phase transition. Most of these works give the violation of the intermittency patterns but show remarkable scaling behaviors between $F_{q}$ and $F_{2}$, and there seems to exist a universal exponent $\nu^{[1,3,6]}$. It is suggested that the exponent $\nu$ can be used as a useful diagnostic tool to detect the formation of QGP. In $[4,5] \ln F_{q}$ are studied analytically for first-order phase transition and are expanded as power series of $\delta^{1 / 3}$, and it is shown that the set of experimentally fitted coefficients for $\delta^{1 / 3}$ term can be used as a criterion for the onset and the order of the phase transition.

It is known for a long time that the investigation of multiplicity fluctuations is very different in heavy-ion collisions, though the power-law dependence of $\ln F_{q}$ on $\delta, F_{q} \propto \delta^{-\varphi_{q}}$, has been found ubiquitous in hadronic and leptonic processes $^{[7]}$. The main differences between heavy-ion physics and hadronic \& leptonic ones on multiplicity fluctuations were noticed earlier in Ref. [8]. In Ref. $[9,10]$ an alternative way was proposed to study the fluctuations by means of factorial moments of the multiplicity difference (FMMD) between two well-separated bins. This alternative is a hybrid of the usual factorial correlators ${ }^{[11]}$ and wavelets ${ }^{[12]}$ because $W_{j k}$ in Haar wavelet analysis is just the difference of multiplicities in two nearest bins. When discussing the multiplicity difference, the two bins are not necessary the nearest ones. Instead, it will be assumed in present study that the two bins are well-separated. Let the two bins, each of size $\delta^{2}$ and separated by $\Delta$, have multiplicities $n_{1}$ and $n_{2}$, and define their multiplicity difference $m=\left|n_{1}-n_{2}\right|$. Let $Q_{m}$ be the distribution of multiplicity difference, which may be dependent on $\Delta, \delta$ and details of the process. Scaled FMMD are defined as

$$
\mathcal{F}_{q}=f_{q} / f_{1}^{q}, \quad f_{q}=\sum_{m} m(m-1) \cdots(m-q+1) Q_{m},
$$

Moments defined above are similar to but not the same as the Bialas-Peschanski correlators ${ }^{[11]} F_{q_{1} q_{2}}$, because of (1) $\mathcal{F}_{q}$ are moments of the multiplicity difference between two bins and $(2) \mathcal{F}_{q}$ may depend on both $\Delta$ and $\delta$ while $F_{q_{1} q_{2}}$ depends only on $\Delta$.

In Ref. [9], $\mathcal{F}_{q}$ are numerically studied within Ginzburg-Landau model. The scaling behaviors between $\mathcal{F}_{q}$ and $\mathcal{F}_{2}, \mathcal{F}_{q} \propto \mathcal{F}_{2}^{\beta_{q}}$, are shown with $\beta_{q}=(q-1)^{\gamma}$ and a universal exponent $\gamma=1.099$. In Ref. [10], $\mathcal{F}_{q}$ are analytically studied for very small $\delta$ within the same model for second-order phase transition. The dynamical components of the moments are introduced and are shown to have similar scaling behaviors. The universal exponent $\gamma=1.3424$ given in [10] for dynamical fluctuations is shown to be different from that in [9] but close to $\nu$ given in the study of usual factorial moments ${ }^{[3,6]}$. The closeness of $\gamma=1.3424$ to $\nu \simeq 1.30$ is reasonable because they both describe dynamical fluctuations in phase transition. The little difference comes from the different $x$ regions concerned: $\nu$ corresponds to $x$ region around $-\ln x \sim 1$ but $\gamma$ to $x \rightarrow 0$, as discussed in [10]. The most important feature about this $\gamma$ is that it is completely determined by the general features of the model and independent of the parameters for the model.

In this Letter, scaled FMMD $\mathcal{F}_{q}$ in first-order phase transition will be studied analytically for very small bin size $\delta$. For simplicity, the discussion is limited to two identical small bins under the condition that the mean multiplicity in each bin is less than or equal to 1.0.

As a base and starting point, discuss the trivial and simplest case in which there is no correlations between the two bins and within each bin. Let the mean multiplicity in each bin is $s$. Because no dynamical reason is assumed, the 
multiplicity distribution for each bin is a Poisson one

$$
P_{n}(s)=\frac{s^{n}}{n !} \exp (-s)
$$

From this distribution, one can deduce the multiplicity difference distribution as

$$
P_{m}(s)=I_{m}(2 s) e^{-2 s}\left(2-\delta_{m 0}\right)
$$

where $I_{m}(z)$ is the modified Bessel function of order $m$,

$$
I_{m}(z)=\sum_{k=0}^{\infty} \frac{(z / 2)^{2 k+m}}{k !(k+m) !}
$$

FMMD for pure statistical fluctuations are

$$
f_{q}^{\text {(stat })}=\sum_{m \geq q} m(m-1) \cdots(m-q+1) P_{m}(s) .
$$

To complete the summation in last equation, one can introduce a generating function ${ }^{[9]}$

$$
G(x, s)=2 e^{-2 s} \sum_{m=0}^{\infty} x^{m} I_{m}(2 s), \quad G_{q}(x, s)=\frac{d^{q} G(x, s)}{d x^{q}}
$$

With this function, $f_{q}^{(\text {stat })}$ can be rewritten as

$$
f_{q}^{(\text {stat })}=G_{q}(1, s) \equiv G_{q}(s)
$$

Direct algebra shows that

$$
G(x, s)=2 e^{(x-2) s}\left[a_{0}^{0}+\sum_{i=1}^{\infty} a_{i}^{0} \frac{d^{i}}{d x^{i}} \frac{1-\exp (-x s)}{x}\right]
$$

with $a_{i}^{0}=(-1)^{i} s^{2 i} /(i !)^{2}$ for $i=0,1, \cdots$, and that

$$
G_{q}(s)=2 e^{-s}\left[a_{0}^{q}+\sum_{i=1}^{\infty} a_{i}^{q} \sum_{j=i}^{\infty} \frac{(-1)^{j} s^{j+1}}{(j+1)(j-i) !}\right]
$$

where $a_{i}^{q}$ can be calculated by recurrence relation from $a_{i}^{0}, a_{0}^{q}=s a_{0}^{q-1}, a_{1}^{q}=s a_{1}^{q-1}, a_{i}^{q}=s a_{i}^{q-1}+a_{i-1}^{q-1},(i \geq 2)$.

In this Letter, we are interested only in small bin analysis, for which the mean multiplicity is less than 1 . Then $G_{q}(s)$ can be approximately written as

$$
G_{q}(s)=2 \mathrm{e}^{-s} s^{q}\left[1+\frac{s^{4}}{(q+1)(q+2)}-\frac{2 s^{5}}{(q+1)(q+2)(q+3)}+\frac{3 s^{6}}{(q+1)(q+2)(q+3)(q+4)}\right]
$$

and all other terms can be omitted. Direct estimation shows that the error caused by this approximation is less than $1 \%$ for $s \leq 1.0$.

For cases with dynamical fluctuations due to phase transition, the distribution of multiplicity difference is ${ }^{[9]}$

$$
Q_{m}(\delta, \tau)=Z^{-1} \int \mathcal{D} \phi P_{m}\left(\delta^{2} \tau|\phi|^{2}\right) e^{-F[\phi]}
$$

where $\tau$ is an indication of lifetime of the whole parton system, $\mathcal{D} \phi=\pi d|\phi|^{2}, Z=\int \mathcal{D} \phi e^{-F[\phi]}$ and the free energy $F[\phi]=\int_{\delta^{2}} d z\left[a|\phi|^{2}+b|\phi|^{4}+c|\phi|^{6}\right]$ for first-order phase transition.

Substituting $Q_{m}(\delta, \tau)$ into Eq. (1), one gets

$$
f_{q}=\int_{0}^{\infty} d y G_{q}\left(\tau u x^{2} y\right) \mathrm{e}^{-y^{3}+u x^{2} y+x y^{2}} / \int_{0}^{\infty} d y \mathrm{e}^{-y^{3}+u x^{2} y+x y^{2}}
$$


with $x=-b(\delta / c)^{2 / 3}$ related with the bin width $\delta, u=|a| c / b^{2}$. As discussed in Ref. [4-6], $b$ is negative for first-order phase transition. So $x$ and $u$ are both positive in present discussions. For the convenience of analytical calculations, define $^{[4]}$

$$
H_{q}(u, v)=\int_{0}^{\infty} y^{q} \exp \left(-y^{3}+u v^{2} y+v y^{2}\right)
$$

which satisfies recurrence relations

$$
\begin{aligned}
H_{2}(u, v) & =\frac{1}{3}+\frac{1}{3}\left(u v^{2} H_{0}(u, v)+2 v H_{1}(u, v)\right), \\
H_{q+3}(u, v) & =\frac{1}{3}\left[(q+1) H_{q}(u, v)+u v^{2} H_{q+1}(u, v)+2 v H_{q+2}(u, v)\right] .
\end{aligned}
$$

With $H_{q}(u, v), f_{q}$ can be expressed as

$$
f_{q}=\frac{2}{H_{0}(u, x)}\left[H_{q}+\frac{\left(\tau u x^{2}\right)^{4} H_{q+4}}{(q+1)(q+2)}-\frac{2\left(\tau u x^{2}\right)^{5} H_{q+5}}{(q+1)(q+2)(q+3)}+\frac{3\left(\tau u x^{2}\right)^{6} H_{q+6}}{(q+1)(q+2)(q+3)(q+4)}\right] .
$$

all $H_{q}$ in the bracket in last equation should be read as $H_{q}(-(\tau-1) u, x)$.

The scaled FMMD $\mathcal{F}_{q}$ obtained contain contributions from statistical fluctuations, contrary to the usual scaled factorial ones. One can see this clearly if one notices the fact that $\mathcal{F}_{q}$ are not equal to 1 for the case with pure statistical fluctuations. To eliminate the pure fluctuations from the moments, one can define the dynamical scaled FMMD as ${ }^{[10]}$

$$
\mathcal{F}_{q}^{\text {(dyn) }}=\frac{\mathcal{F}_{q}}{\mathcal{F}_{q}^{\text {(stat) }}}
$$

To make the definition sense, one should ensure that the mean multiplicity is the same for all the calculation of the moments concerned. In Ginzburg-Landau model, the mean multiplicity is $\bar{s}=\tau u x^{2} H_{1}(u, x) / H_{0}(u, x)$. Then deviations of $\mathcal{F}_{q}^{(\mathrm{dyn})}$ from one should indicate the existence of dynamical fluctuations. Different from the case for second-order phase transition, $\bar{s} \propto x^{2}$ in small $x$ region for first-order phase transition, thus the pure statistical fluctuations have no contribution to the slopes of $\ln \mathcal{F}_{q}$ in the $x$ region we are now interested in. Thus, we will only discuss $\ln \mathcal{F}_{q}$ in present discussion.

The dependences of $\ln \mathcal{F}_{q}$ on $-\ln x$ from 2.0 to 4.0 are shown in Fig. 1 for $\tau=10.0, u=1.0$ and 5.0. The $-\ln x$ range is chosen from the requirement that $\bar{s}$ is much less than 1.0 for $\tau=10.0$ and $u=5.0$. From this figure, one can see clearly that $\ln F_{q}$ depend strongly on parameters chosen, and $\ln \mathcal{F}_{q}$ decrease quickly with the increase of $-\ln x$ in the $x$ range chosen. For sufficiently large $-\ln x, \ln \mathcal{F}_{q}$ satuate to values independent of $u$. In fact, the satuation values are independent of any parameter in the model.

Scaling behaviors between $\mathcal{F}_{q}$ are shown in Fig. 2 for the same choices of parameters as in Fig. 1. One can see that the scaling behavior, $\mathcal{F}_{q} \propto \mathcal{F}_{2}^{\beta_{q}}$, is valid for either set of the parameters, and the slopes show weak dependence Because of the linearity of curves in Fig. 2 for $\mathcal{F}_{q}, \beta_{q}$ can be determined accurately. One can guess that for small $x$, $\beta_{q}$ may be independent of all parameters in the model, and it is indeed the case. For very small $x$, one can get, using $H_{q}(u, v) \simeq \frac{1}{3}\left[\Gamma\left(\frac{q+1}{3}\right)+v \Gamma\left(1+\frac{q}{3}\right)\right]$

$$
\beta_{q}^{(\mathrm{dyn})}=\frac{(q-1) a_{0,1} / a_{0,0}+a_{q, 1} / a_{q, 0}-q a_{1,1} / a_{1,0}}{a_{0,1} / a_{0,0}+a_{2,1} / a_{2,0}-2 a_{1,1} / a_{1,0}},
$$

with $a_{q, 0}=\Gamma\left(\frac{q+1}{3}\right)$ and $a_{q, 1}=\Gamma\left(1+\frac{q}{3}\right)$. $\beta_{q}$ as function of $\ln (q-1)$ is shown in Fig. 3. A perfect scaling behavior is shown

$$
\beta_{q}=(q-1)^{\gamma}
$$

with $\gamma=1.4066$. This exponent corresponds to the limit $x \rightarrow 0$. In real experimental analysis, one fits curves for $\ln \mathcal{F}_{q}$ and $\ln \mathcal{F}_{2}$ at finite $x$, so that one should get an exponent with a little difference from the one given here. In fact, the experimentally obtained exponent should be less than 1.4066 .

It should be pointed out that the exponent can be obtained simply from the leading term in $G_{q}$ by dropping off all non-leading terms because they are related to higher orders of $x$ and have no contribution to $\gamma$ which is connected with properties of the moments in the limit $x \rightarrow 0$. The exponent given here is different from that in [10] for second-order 
phase transition. The main reason is due to the introduction of $|\phi|^{6}$ term in the free-energy. One can see that $|\phi|^{6}$ term is necessary for the study of first-order phase transition. In the case of second-order phae transition, the same term may also play a role. Only when $-x \gg 1$ can the $-y^{3}$ term be neglected in Eq. (11), and one returns to the Ginzburg-Landau description of the phase transition studied in [3]. So the discussions in this Letter are different from those in former studies. In fact, if $|\phi|^{6}$ term plays an important role, $\gamma$ should be close to 1.4066 for both first-order and second-order phase transitions in small $\delta$ analyses. With the increase of the importance of $|\phi|^{6}$ term, the exponent $\gamma$ for second-order phase transition can undergo an increase from 1.3424 in [10] to 1.4066 given here. But if there is no phase transition, the exponent $\gamma$ should be much less than 1.3424. Thus if an exponent $\gamma$ for the dynamical fluctuations is found near to 1.4, then the occurrence of quark-hadron phase transition can be pronounced.

In summary, scaled FMMD are studied analytically within Ginzburg-Landau model in a kinetical region with mean multiplicity in single bin less than 1.0 for first-order quark-hadron phase transition. The dynamical fluctuations in FMMD are extracted, which give the same physical contents as the usual scaled factorial moments. Scaling behaviors between scaled FMMD are shown, and a truly universal exponent $\gamma=1.4066$ is given.

This work was supported in part by the NNSF, the SECF and Hubei NSF in China. One of the authors (C.B. Yang) is grateful for fruitful discussions with Prof. R.C. Hwa.

[1] A. Bialas and R.C. Hwa, Phys.Lett. B253, 436(1991); R.C. Hwa and M.T. Nazirov, Phys. Rev. Lett. 69, 741(1992).

[2] R.C. Hwa and J.C. Pan, Phy. Rev. D45, 106(1992).

[3] R.C. Hwa, Phys. Rev. D47, 2773(1993); R.C. Hwa and J.C. Pan, Phys. Lett. B297, 35(1992).

[4] X. Cai, C.B. Yang, and Z.M. Zhou, Phys. Rev. C54, 2775(1996).

[5] C.B. Yang, X.R.Wang and X. Cai, Science in China, 27, 624(1997)(in Chinese); 40, 1065(1997) (in English).

[6] R.C. Hwa, Phys. Rev. C50, 383(1994); I.A. Lebedev and M.T. Nazirov, Mod. Phys. Lett. A9, 2999(1994); A.K. Mohanty and

S.K. Kataria, Phys. Rev. Lett. 73, 2672(1994); L.F. Babichev, D.V. Klenitsky, V.I. Kuvshinov, Phys. Lett. B345, 269(1995).

[7] E.A. DeWolf, I.A. Dremin, and W. Kittel, Phys. Rep. 270, 1(1996).

[8] I. Sarcevic, in Fluctuations and Fractal Structure, proceedings of Ringberg Workshop, Rinhberg Castle, Germany 1991, edited by R.C. Hwa, W. Ochs, and N. Schmitz (World Scientific, Singapore, 1992); H.-T. Elze and I. Sarcevic, Phys. Rev. Lett. 68, 1988(1992); H.C. Eggers, H.-T. Elze and I. Sarcevic, Int. J. Mod. Phys. A9, 3821(1994).

[9] R.C. Hwa, Preprint OITS631, hep-ph/9707272, submitted to Phys. Rev. D.

[10] C.B. Yang and X. Cai, hep-ph/9710517, submitted to Phys. Rev. C.

[11] A. Bialas and R. Peschanski, Nucl. Phys. B273, 703(1986); B308, 857(1988).

[12] M. Gell-Mann and M. Levy, Nuovo Cimento 16, 53(1960).

\section{Figure Captions}

Fig. 1 Dependences of $\ln \mathcal{F}_{q}$ on $-\ln x$ for $\tau=10.0, u=1.0$ and $u=5.0$. From lower to upper are curves for $q=2,3,4,5,6,7,8,9$, respectively.

Fig. 2 Scaling behaviors of $\ln \mathcal{F}_{q}$ vs $\ln \mathcal{F}_{2}$ for the same choices of parameters as in Fig.1. From lower to upper are curves for $q=3,4,5,6,7,8,9$, respectively.

Fig. 3 Scaling behavior of $\ln \beta_{q}$ vs $\ln (q-1)$. 


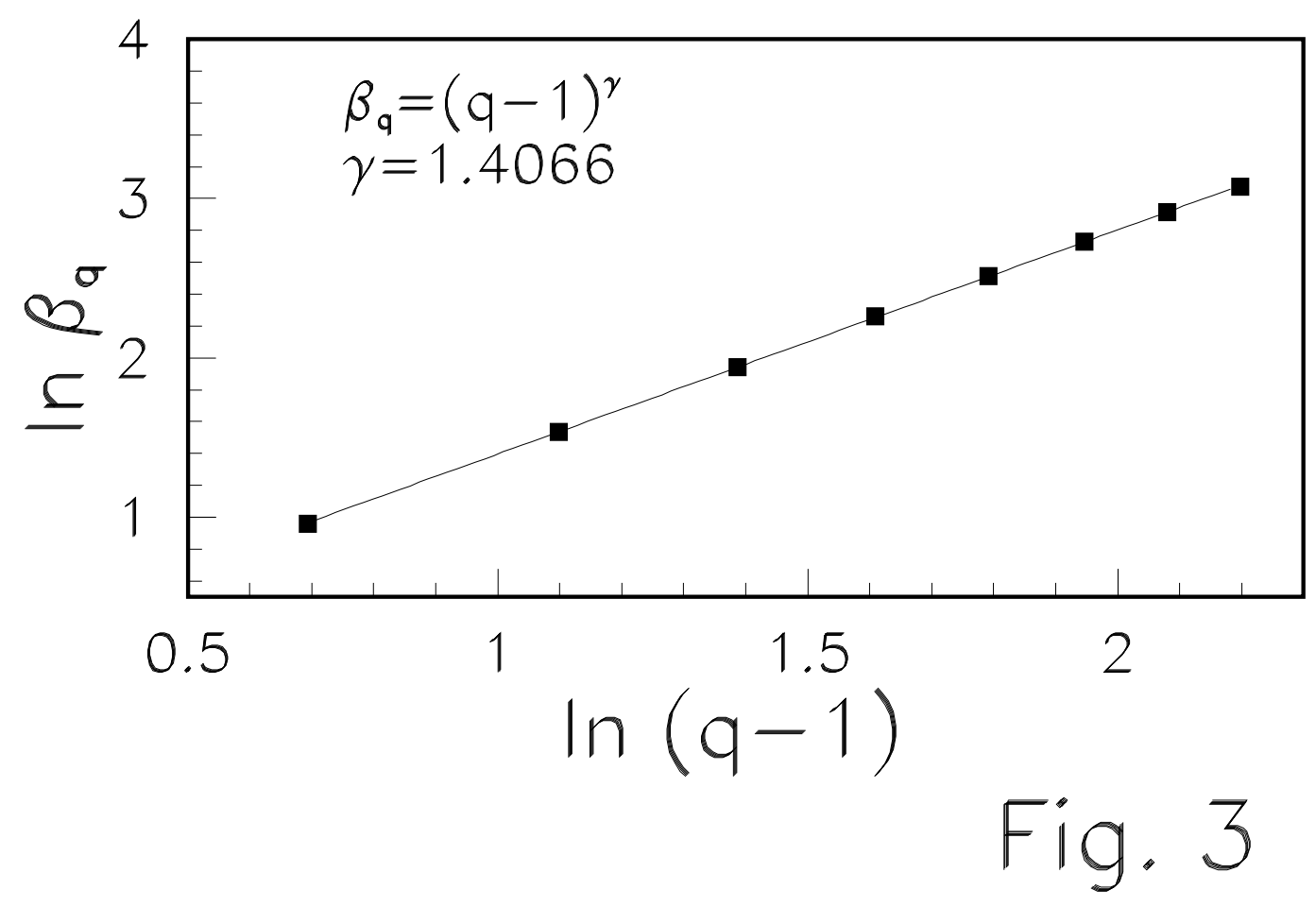




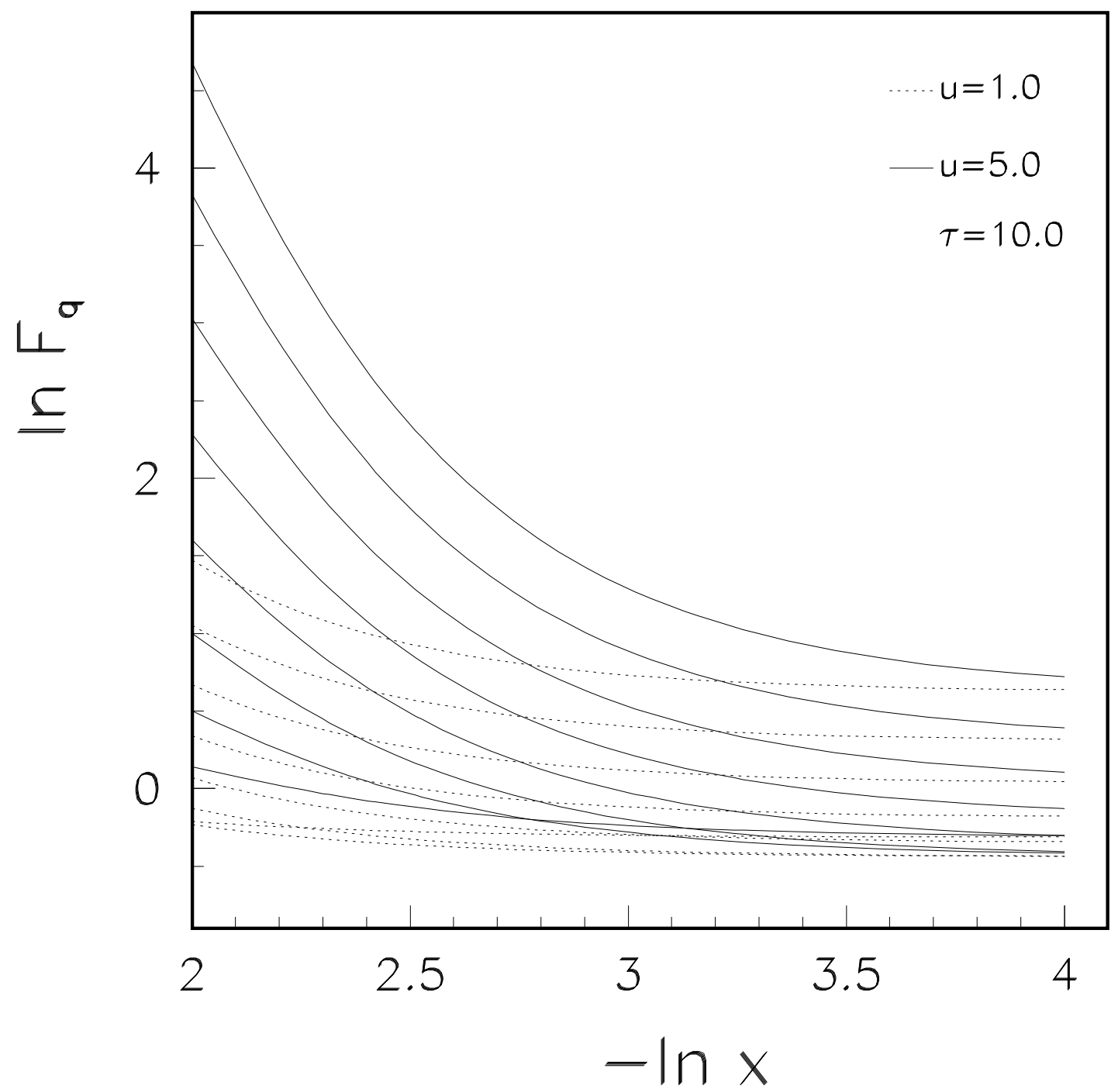

Fig. 1 


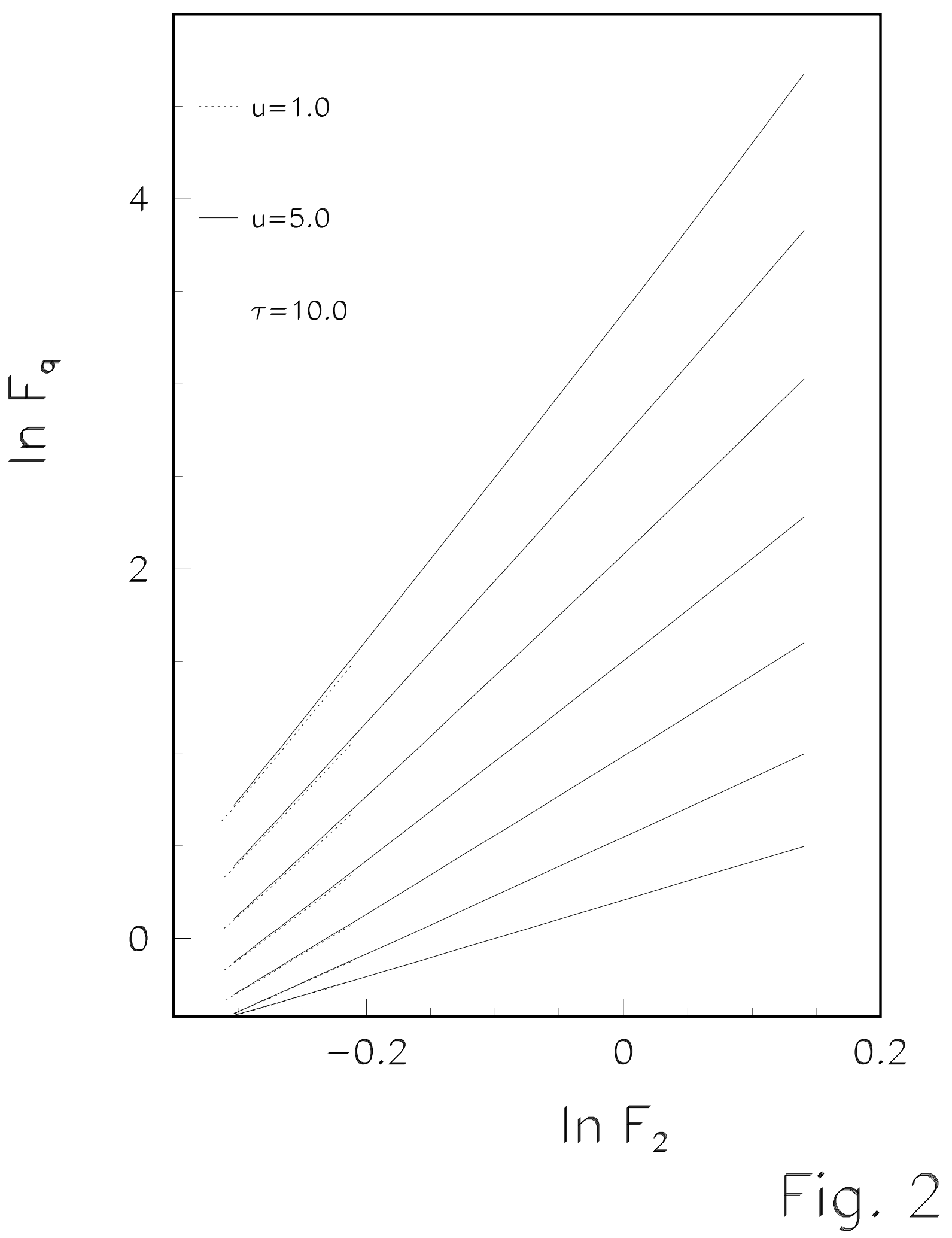

